\title{
Therapy sequencing strategies in multiple myeloma: who, what and why?
}

\author{
Caitlin Costello*,1 \& Joseph R Mikhael ${ }^{2}$ \\ ${ }^{1}$ Department of Medicine, Moores Cancer Center, University of California, San Diego, La Jolla, CA 92093, USA \\ ${ }^{2}$ Department of Medicine, Mayo Clinic of Arizona, Phoenix, AZ 85054, USA \\ * Author for correspondence: ccostello@ucsd.edu
}

First draft submitted: 28 September 2017; Accepted for publication: 5 October 2017; Published online: 8 December 2017

Keywords: drug sequencing • multiple myeloma • novel therapy

The past decade has witnessed a boom in the therapeutic landscape for the management of multiple myeloma. Improvement in the depth of response with novel combinations has resulted in a near doubling of the overall survival (OS) among patients with multiple myeloma (MM) [1,2]. Several new agents, including the third-generation immunomodulatory agent (IMiD) - pomalidomide, the second-generation proteasome inhibitors (PIs) - carfilzomib and ixazomib, the histone deacetylaste inhibitor - panobinostat, and two monoclonal antibodies - elotuzumab and daratumumab, have all been recently approved. The multitude of novel agents and combinations, however, has resulted in somewhat of a conundrum for physicians who manage myeloma patients - how do we incorporate these new treatments into clinical practice? While there is no ideal sequence of therapy for every patient, there are several nuances worthy of discussion to help clarify the therapeutic options for each stage of treatment.

\section{Goals of treatment}

The evaluation of agents in novel combinations has shown the importance of depth of response, consistently correlating with higher complete remission (CR) rates and progression-free survival (PFS). It is now possible to achieve a high level of tumor burden reduction leading to negative minimal residual disease, an important goal in the treatment of myeloma that is associated with prolonged PFS and possibly longer OS [3,4]. This information reinforces the objective that while antimyeloma therapy should be chosen carefully for every individual patient based on disease characteristics, age, frailty and co-morbidities, the primary goal of therapy is to improve OS.

\section{Induction therapy}

The approach to the treatment of newly diagnosed MM has more recently centered around two questions: doublet or triplet drug combinations; and upfront or delayed autologous stem-cell transplant (ASCT). The SWOG S0777 study demonstrated the importance of adding bortezomib to lenalidomide and dexamethasone (RVD) as a triplet combination when compared with the doublet combination of lenalidomide and dexamethasone alone (Rd) [5]. The use of RVD showed an improvement in overall response rates (ORR: 82 vs 72\%), median PFS (55 vs 43 months; $\mathrm{p}=0.0037)$ and OS (75 vs 64 months; $\mathrm{p}=0.25)$ in those patients receiving the triplet compared with those in the doublet arm. The Integroupe Francophone du Myeloma (IFM) 2013-04 study showed the superiority of a PI/IMiD combination when bortezomib/thalidomide/dexamethasone (VTD) was compared with bortezomib/cyclophosphamide/dexamethasone (VCD) and found to be superior with an overall response rate in the VTD arm of 92.3 versus $83.4 \%$ in the VCD arm [6]. Based on response rates, depth of response and PFS as surrogate markers for outcome, three-drug combinations including an IMiD, PI and dexamethasone are currently the standard of care for induction.

\section{High-dose therapy \& ASCT}

Despite improved induction strategies, ASCT remains the standard of care in eligible patients. This was confirmed by the recently published trial by the IFM in which all patients underwent induction with the RVD triplet for three cycles followed by randomization to consolidation therapy with either RVD for five more cycles or with high-dose melphalan plus ASCT followed by RVD consolidation for two cycles. Patients in both arms received 1 year of

Future : Medicine 
lenalidomide maintenance. The median PFS was significantly longer in the ASCT group than in the group that received RVD alone (50 vs 36 months; hazard ratio [HR]: 0.65; p < 0.001), however, the OS at 4 years did not differ significantly between the two groups ( 81 and $82 \%$, respectively) [7]. This transplant trial confirmed the findings of other published studies that address the role of ASCT [8,9]. The role of salvage ASCT is an important consideration as well. In the IFM study, $79 \%$ of patients who received RVD alone subsequently received second-line therapy followed by salvage transplantation, proving the feasibility of this strategy for patients who choose to defer upfront ASCT [7].

\section{Treatment for relapsed disease}

The choice of the appropriate combination of agents at relapse can be challenging due to the growing list of available therapeutic options. The field of myeloma has significantly changed with the use of more combination therapy as opposed to a more sequential approach. This has led to the $\mathrm{A}+\mathrm{B}$ combination is superior to $\mathrm{A}$ than $\mathrm{B}$. In addition to this principle, one must consider the value of retreatment - if a patient has tolerated a regimen previously with reasonable depth and duration of response (at least 6 months), then retreatment should be considered.

Multiple considerations must be made when making a decision on the next regimen, and these include seven major factors:

\section{Prior maintenance therapy}

Patients who have relapsed while receiving bortezomib- or lenalidomide-based maintenance therapy are deemed to be refractory and it is prudent to switch drug classes when choosing the next regimen. A patient who has previously responded to a given agent without major toxicity can be retreated at relapse with the same agent or with an agent of the same class as its backbone [10]. The CASTOR and POLLUX studies compared triplets including daratumumab to the standard $\mathrm{Vd}$ and $\mathrm{Rd}$ and have shown dramatic superiority in the daratumumab-containing regimens $[11,12]$. These combinations could be used in relapsed patients who are bortezomib- or lenalidomidesensitive, respectively. Similarly, the Aspire trial documented the improved ORR and PFS in patients receiving carfilzomib in combination with Rd as opposed to Rd alone [13]. This pivotal trial is the first randomized study to demonstrate the superiority and the tolerability of a PI and IMiD combination in relapsed disease. For the scenario where patients may be resistant to both lenalidomide and bortezomib at first relapse, second-generation IMiDs or PIs have a role. Pomalidomide has activity in double refractory patients $[14,15]$ and can be used in combination with daratumumab or carfilzomib $[16,17]$.

Rate of relapse \& risk status

The rate of relapse and risk status of disease can help determine both the timing of treatment initiation and the aggressiveness of the approach. Symptomatic relapse requires a more rapid and aggressive approach with combination therapy to relieve symptoms and to avoid further organ damage [10]. Triplet combinations are critical in this scenario, however, when there is an urgent need for cytoreduction, a salvage infusional regimen combining conventional cytotoxic agents including etoposide, cisplatin, cyclophosphamide and dexamethasone can be used [18].

The approach to slow biochemical progression however can allow for a watch-and-wait strategy that may not require urgent initiation of therapy, suggesting a more indolent form of disease.

\section{Co-morbidities}

Co-morbidities and adverse events of prior treatments can help guide therapy decisions based on an interest in avoiding specific potential toxicities of next-line therapy. The choice between PIs requires careful attention to peripheral neuropathy and cardiac co-morbidities. While the incidence of peripheral neuropathy with carfilzomib is lower than with bortezomib, the greater risk for carfilzomib-related cardiotoxicity (hypertension, cardiac failure or ischemic heart disease) must be carefully considered [19]. Lenalidomide is excreted through the kidneys and thus requires dose adjustments according to the degree of renal insufficiency [20]. The risk of myelosuppression must also be considered when considering an IMiD, particularly in patients with cytopenias [21,22].

\section{Frailty}

The benefit of therapy may be outweighed by the increased toxicity in the frail patient, oftentimes leading to treatment interruptions and reduced efficacy. Dose reduction is important to balance disease control with excess 
toxicity. The dosing, schedule and route of administration can make substantial differences in the safety profile of therapy and thereby allow for more sustained durations of therapy.

\section{Availability}

The modern treatment of myeloma is expensive and many options for treatment are not yet available worldwide. Accessibility is a realistic consideration when choosing therapy depending on insurance coverage and countryspecific healthcare systems.

\section{Convenience}

Long-term treatment has emerged as a standard of care with goals to provide continuous disease suppression, deepened responses and prolonged survival. Patient quality of life, particularly in regard to the tolerability and convenience of therapy, has become a focus of attention. All-oral treatment combinations can be an attractive option for patients who have limited ability to travel to clinic visits, including those who may live far away or frail/elderly patients. Combinations such as ixazomib/lenalidomide/dexamethasone [23] or cyclophosphamide/pomalidomide/dexamethasone [24] are two examples of convenient all-oral regimens for patients.

\section{Clonal evolution}

Multiple relapses and clonal evolution may lead to the acquisition and accumulation of new genetic alterations and clonal heterogeneity, leading to an increasingly aggressive phenotype $[25,26]$. These secondary mutations alter the patient's risk stratification, have an additional effect on prognosis [27] and may have therapeutic implications. Clinically, it is important to recognize the phenomenon of free light chain escape or new renal dysfunction as an indicator of clonal evolution. As additional clones emerge, therapy is most likely to be effective if it targets the dominant mutation in the clone rather than a subclone. A combination therapy approach however provides a strong biologic rationale to eradicate all clones and avoid the selection of aggressive ones. Importantly, retreatment of a patient with a particular agent may be considered if a sensitive subclone reemerges during the disease course.

\section{Second relapse \& beyond}

Given the efficacy of triplet regimens and the growing number of upcoming trials with three- or even four-drug regimens, triplet regimens have become the standard of care. It is important however to understand that many of the triplet studies using lenalidomide as a backbone limited the enrollment to lenalidomide-sensitive or -naive patients, and may not be directly applicable to clinical practice. For patients in second relapse or beyond, any of the prior combinations not tried after first relapse are all viable options. The choice of treatment for the multiple relapsed patients should abide by the same considerations as discussed above. While triplet-based combinations are preferred, doublets can offer significant responses for patients in whom there are concerns for toxicities with three drugs. Pomalidomide/dexamethasone [21] and daratumumab monotherapy [28] are well-tolerated regimens that offer meaningful response rates.

\section{Future perspective}

The future of therapy for multiple myeloma remains bright. Novel drugs on the horizon include selinexor (nuclear exporter inhibitor), venetoclax (Bcl2-inhibitor), check-point inhibitors, bispecific T-cell engager antibodies and chimeric antigen receptor $\mathrm{T}$ cells, all of which will further enhance the treatment landscape for multiple myeloma.

\section{Financial \& competing interests disclosure}

C Costello has received honoraria from Celgene, Takeda and Janssen. JR Mikhael has received institutional research funding from Abbvie, Celgene and Sanofi. The authors have no other relevant affiliations or financial involvement with any organization or entity with a financial interest in or financial conflict with the subject matter or materials discussed in the manuscript apart from those disclosed.

No writing assistance was utilized in the production of this manuscript.

\section{References}

1 Kumar SK, Dispenzieri A, Lacy MQ et al. Continued improvement in survival in multiple myeloma: changes in early mortality and outcomes in older patients. Leukemia 28(5), 1122-1128 (2014). 
2 Rajkumar V. Myeloma today: disease definitions and treatment advances. Am. J. Hematol. 91(9), 965 (2016).

3 Kumar S, Paiva B, Anderson KC et al. International Myeloma Working Group consensus criteria for response and minimal residual disease assessment in multiple myeloma. Lancet Oncol. 17(8), e328-e346 (2016).

4 Martinez-Lopez J, Lahuerta JJ, Pepin F et al. Prognostic value of deep sequencing method for minimal residual disease detection in multiple myeloma. Blood 123(20), 3073-3079 (2014).

5 Durie BG, Hoering A, Abidi MH et al. Bortezomib with lenalidomide and dexamethasone versus lenalidomide and dexamethasone alone in patients with newly diagnosed myeloma without intent for immediate autologous stem-cell transplant (SWOG S0777): a randomised, open-label, Phase 3 trial. Lancet 389(10068), 519-527 (2017).

6 Moreau P, Hulin C, Macro M et al. VTD is superior to VCD prior to intensive therapy in multiple myeloma: results of the prospective IFM2013-04 trial. Blood 127(21), 2569-2574 (2016).

7 Attal M, Lauwers-Cances V, Hulin C et al. Lenalidomide, bortezomib, and dexamethasone with transplantation for myeloma. N. Engl. J. Med. 376(14), 1311-1320 (2017).

8 Gay F, Oliva S, Petrucci MT et al. Chemotherapy plus lenalidomide versus autologous transplantation, followed by lenalidomide plus prednisone versus lenalidomide maintenance, in patients with multiple myeloma: a randomised, multicentre, Phase 3 trial. Lancet Oncol. 16(16), 1617-1629 (2015).

9 Cavo M, Pantani L, Pezzi A et al. Bortezomib-thalidomide-dexamethasone (VTD) is superior to bortezomib-cyclophosphamide-dexamethasone $(\mathrm{VCD})$ as induction therapy prior to autologous stem cell transplantation in multiple myeloma. Leukemia 29(12), 2429-2431 (2015).

10 Laubach J, Garderet L, Mahindra A et al. Management of relapsed multiple myeloma: recommendations of the International Myeloma Working Group. Leukemia 30(5), 1005-1017 (2016).

11 Palumbo A, Chanan-Khan A, Weisel K et al. Daratumumab, bortezomib, and dexamethasone for multiple myeloma. N. Engl. J. Med. 375(8), 754-766 (2016).

12 Dimopoulos MA, Oriol A, Nahi H et al. Daratumumab, lenalidomide, and dexamethasone for multiple myeloma. N. Engl. J. Med. 375(14), 1319-1331 (2016).

13 Stewart AK, Rajkumar SV, Dimopoulos MA et al. Carfilzomib, lenalidomide, and dexamethasone for relapsed multiple myeloma. $N$. Engl. J. Med. 372(2), 142-152 (2015).

14 Lacy MQ, Allred JB, Gertz MA et al. Pomalidomide plus low-dose dexamethasone in myeloma refractory to both bortezomib and lenalidomide: comparison of 2 dosing strategies in dual-refractory disease. Blood 118(11), 2970-2975 (2011).

15 Leleu X, Attal M, Arnulf B et al. Pomalidomide plus low-dose dexamethasone is active and well tolerated in bortezomib and lenalidomide-refractory multiple myeloma: Intergroupe Francophone du Myélome 2009-02. Blood 121(11), 1968-1975 (2013).

16 Chari A, Suvannasankha A, Fay JW et al. Daratumumab plus pomalidomide and dexamethasone in relapsed and/or refractory multiple myeloma. Blood 130(8), 974-981 (2017).

17 Shah JJ, Stadtmauer EA, Abonour R et al. Carfilzomib, pomalidomide, and dexamethasone for relapsed or refractory myeloma. Blood 126(20), 2284-2290 (2015).

18 Griffin PT, Ho VQ, Fulp W et al. A comparison of salvage infusional chemotherapy regimens for recurrent/refractory multiple myeloma. Cancer 121(20), 3622-3630 (2015).

19 Dimopoulos MA, Goldschmidt H, Niesvizky R et al. Carfilzomib or bortezomib in relapsed or refractory multiple myeloma (ENDEAVOR): an interim overall survival analysis of an open-label, randomised, Phase 3 trial. Lancet Oncol. 18(10), 1327-1337 (2017).

20 Dimopoulos MA, Palumbo A, Attal M et al. Optimizing the use of lenalidomide in relapsed or refractory multiple myeloma: consensus statement. Leukemia 25(5), 749-760 (2011).

21 San Miguel J, Weisel K, Moreau P et al. Pomalidomide plus low-dose dexamethasone versus high-dose dexamethasone alone for patients with relapsed and refractory multiple myeloma (MM-003): a randomised, open-label, Phase 3 trial. Lancet Oncol. 14(11), 1055-1066 (2013).

22 Dimopoulos M, Spencer A, Attal M et al. Lenalidomide plus dexamethasone for relapsed or refractory multiple myeloma. N. Engl. J. Med. 357(21), 2123-2132 (2007).

23 Moreau P, Masszi T, Grzasko N et al. Oral ixazomib, lenalidomide, and dexamethasone for multiple myeloma. N. Engl. J. Med. 374(17), 1621-1634 (2016).

24 Baz RC, Martin TG, Lin HY et al. Randomized multicenter Phase 2 study of pomalidomide, cyclophosphamide, and dexamethasone in relapsed refractory myeloma. Blood 127(21), 2561-2568 (2016).

25 Keats JJ, Chesi M, Egan JB et al. Clonal competition with alternating dominance in multiple myeloma. Blood 120(5), 1067-1076 (2012).

26 Egan JB, Shi CX, Tembe W et al. Whole-genome sequencing of multiple myeloma from diagnosis to plasma cell leukemia reveals genomic initiating events, evolution, and clonal tides. Blood 120(5), 1060-1066 (2012). 
27 Binder M, Rajkumar SV, Ketterling RP et al. Occurrence and prognostic significance of cytogenetic evolution in patients with multiple myeloma. Blood Cancer J. 6, e401 (2016).

28 Lokhorst HM, Plesner T, Laubach JP et al. Targeting CD38 with daratumumab monotherapy in multiple myeloma. N. Engl. J. Med. 373(13), 1207-1219 (2015). 
